is not pushed down into the position represented in the illustration. The apparatus is next sterilised at a temperature of 150 to $170^{\circ} \mathrm{C}$. The plug is withdrawn, and the medium is poured into the flask by means of a funnel with a long stem. Care must be taken that the neck of the flask does not become soiled with the medium in doing so. The plug is replaced, and the medium in the interior of the flask sterilised by the usual means.

Having proceeded so far, the free extremity of the cotton plug is cut off, singed, and pushed down into position. The mercury (M) is next poured into the space above the plug. That in the bend of the tube $(m)$ is not introduced till afterwards.

The apparatus is now ready for the deoxygenation of the medium, which, if gelatinised, should be melted in a warm bath. From 2 to 3 per cent. of grape sugar should have been added to it previous to its being placed in the flask. The stream of hydrogen is passed through it by attaching the end of the glass tube $(\mathrm{G})$ to an india-rubber tube connected with a cylinder of compressed hydrogen. The hydrogen is purified by being passed through solutions of potash and pyrogallic acid. The escape gas readily bubbles through the mercury in the neck. The medium is liquefied by placing the bulb of the flask in warm water. After the gas has continued to pass for something like a quarter to half an hour, the supply is cut off, the end of the tube drawn up, and the medium is allowed to solidify by immersing the bulb in an ice bath. Any contraction which takes place in the atmosphere of hydrogen as it is cooling can be compensated by allowing a little more gas to enter. Indeed the gas may be allowed to stream through the flask during the whole time in which solidification is taking place, provided that the end of the tube is pulled upwards.

The medium is thus deprived of its oxygen, and can now be inoculated. Suppose, in the first place, that it is desired to make a surface culture. The gas supply is cut off at $\mathrm{G}$, the mercury is decanted from the neck of the flask, and the plug with the tube is withdrawn by means of a hook. The silk cords surrounding the plug aid in so doing by being readily caught by the hook. The inoculation is effected by means of an ordinary platinum needle, and immediately thereafter the cotton plug is replaced, and the mercury again poured into the space above it. A thin layer of glycerine is run over the surface of the mercury, and the remaining part of the neck of the flask is filled with melted paraffin, which is allowed to solidify. The paraffin fixes the tube and prevents the mercury from running out when the flask is rotated, as afterwards described. A drop or two of glycerine is introduced into the tube at $\mathrm{G}$, and the bend $(m)$ is filled with mercury.

This being accomplished, the flask is ready for filling with hydrogen. Its neck is held by a clamp capable of being rotated and the connection with the gas supply at $\mathrm{G}$ is reestablished. A small piece of the paraffin plug is cut out as represented at $c$, and a little of the mercury may be allowed to escape. The flask is now rotated backwards, that is to say, in a direction to the left of the scheme, within a compass of a little more than $45^{\circ}$. The result is that the mercury comes to occupy the position of the dotted line o, and so leaves part of the cotton plug exposed. Through this exposed part the gas escapes. Indeed, it is not necessary to expose the plug entirely, for the gas readily streams through the thin layer of mercury which may happen to cover it. The mercury $(m)$ in the bend of the tube at the same time falls backwards into the bulb (B), leaving the supply tube open. The gas supply is now turned on, and escapes readily through the cotton plug in the neck of the flask. After the gas has streamed through sufficiently long, say, a quarter of an hour, the supply is cut off, and the flask turned round so as again to occupy the upright position, the result being that the mercury in the bulb $(\mathrm{B})$ returns to the bend of the tube $(m)$, and the cotton plug becomes completely covered by that in the neck of the flask. The apertures of entrance and exit are thus simultaneously and instantaneously sealed, and the gas is prevented from escaping. Fresh paraffin is poured into the vacuity left by part having been cut out.

If it is desired to allow the organism to grow all through the medium, inoculation should be practised hefore deoxygenation. The medium can be melted over and over again, and the gas passed through it. When the flask is rotated the medium, if melted, occupies the situation of the dotted line $(v)$, but the bend on the tube brings the orifice into its midst, and causes the gas to pass through it. If the medium be a liquid, the same method of procedure is of course applicable to it.

I have generally renewed the atmosphere of hydrogen week by week. It is questionable, however, whether this is necessary.

Supposing it is desired to examine part of a culture, all that is necessary is to cut round the entire margin of the paraffin and withdraw the plug. The mercury should previously have been run off, both from the neck of the flask and from the tube. After removal of part of the culture the plug and other parts are readjusted, and a stream of gas again passed through the flask.

The advantages possessed by the apparatus are self-evident, and may be summarised as follows :

I. It is of simple construction, and can be made in a few minutes out of ordinary laboratory materials, the cost being thereby reduced to a minimum. ${ }^{1}$

2. The atmosphere of hydrogen can be renewed as often as desired.

3. The escape of gas is prevented by the simplest and best of all valves.

4. It can be employed for surface cultures.

5. From the wide expanse afforded by the medium in the flask it can be used for fractional cultivation, and hence has many of the advantages without the disadvantages of Petri's plates, or other like expedient.

\section{PATHOLOGY OF VACCINIA AND VARIOLA.}

\section{By S. MONCKTON COPEMAN, M.A., M.D.CANTAB.,} M.R.C.P.,

Lecturer on Hygiene and Public IIealth Westminster Hospital Medical School.

BEFORE referring to the most recent work on the pathology of variola in detail, it may be well to briefly recapitulate the subject matter of the paper which I had the honour of bringing before the notice of this Section at the meeting of the Association, held at Bristol in 1894 .

I there stated that the main dispute with reference to the pathology of vaccinia has always centred round the term variolæ vaccinæ, "small-pox of the cow" given to the disease in the first instance by Jenner. Consequently the important question remains as before, whether vaccinia is or is not one with small-pox.

I prefer in this connection, the phrase "one with smallpox," since it matters little from my point of view, whether small-pox on its transference from man to the bovine animal becomes "transmuted" as the phrase is, or is merely modified, as some would maintain. If I were informed on good authority that small-pox and vaccinia are descended both of them from a common stock : from an ancestor for instance which resembled vaccinia far more than it resembled smallpox, I should not be disturbed by the proposition. I should perceive indeed that the seeming vaccinia obtained in the calf by inoculation of small-pox matter into that animal might after all be but a reversion to an antecedent type, and I should be calling to mind a fact of universal experience, namely, that vaccinia-however it may have arisen in the past, or is made to appear in the present-exhibits little tendency to "sport" (as for instance by manifesting a "generalised eruption") in the direction of small-pox. I could understand, I mean, a highly differentiated form reverting, under artificial conditions, to a little differentiated form ; though the reverse process, readily or frequently repeated per saltum would indeed surprise me.

In my former paper, I stated that the problem has been attacked from two separate points of view :-1. Experimental work having for its object the production-if that be possible -in the bovine animal, hy inoculating it with small-pox

1 Mr. Hume, I, Lothian street, Edinburgh, supplies the instrument Rond in the Sortion of pathete. Mediend Associetion, helel in London July-Aurust, r89. 
lymph, of a disease indistinguishable both in its immediate local and in its subsequent general effects, from true vaccinia. 2. Microscopic pathology and bacteriology, including, I may add, the testing of bacteria derived from lymph, also on the bovine animal.

With reference to the first point, I was able to show that of the various ohservers who at different times have worked at this branch of the subject (among whom, of those now living, King, Simpson, Hime, Voigt, Fischer, Haccius, and Eternod, may be mentioned), all, with the exception of Chauveau and his colleagues of the Lyons Commission, claim to have obtained positive results, in a certain number of their experiments, at any rate, as regards the production of typical vaccinia, after one or more removes, as the result of variolation of the cow or calf. In many cases, it is true, a large number of experiments have been abortive, especially among the older observers who made use, for the most part, of heifers or milch cows. Subsequent experience has shown, however. that success is much more likely to be obtained if, instead of heifers or cows, calves be used. On this account, perhaps, Chauveau's want of success may be, in part, explained.

With reference to recent experiments on variolation of the ealf, it is worthy of note also that different observers have obtained local effects in that animal, which in different calves of a series have varied considerably. The final result has, however, after a greater or less number of removes from calf to calf been invariably the same, the production, namely, of a local vesicle indistinguishable by any means at our command, such as the appearance and course there of or the protective power of the lymph derived therefrom, from true vaccinia.

As far as I am aware, the most recent experiments of this nature which have been carried out are those of Dr. Klein and myself, the results of which have been published in full in the reports of the Medical Officer to the Local Government Board and in the Journal of Pathology and Bacteriology respectively. I do not therefore propose on the present occasion to refer to those experiments in detail, but I may perhaps add that, in my own experience at any rate, no question of possible contamination with vaccinia can possibly arise, seeing that all my work was conducted at the Brown Institution, that as a special precaution new scalpels were used, which were invariably first sterilised in a flame; that before and after use the table was thoroughly washed with carbolic acid and water, and during the intervals of use kept exposed to the open air.

As the result of all the work which has been done in this direction, there can, I think, at the present time, be no reasonable doubt as to the possibility of inoculating the calf with variolous matter with the result of producing thereby after one or more removes of a malady which is certainly no longer small-pox as ordinarily understood, seeing that it has now completely lost, when transferred to the human being, its original property of infectiousness. Although this is so, it is nevertheless capable of affording protection, just as vaccinia does, against subsequent vaccination or variolation. In this connection I may perhaps refer to certain experiments of mine on the monkey, which showed that not only is this animal susceptible to inoculation of vaccinia, which runs a course similar to that which is observed in the human being and the calf, but it is equally susceptible to the inoculation of variola. Furthermore, in the case of the monkey, small-pox lymph and vaccine, either human or bovine, appear to possess mutually protective power one against either of the others.

Turning next to the micropatholngy and bacteriology of variolous and more especially of vaccine lympl, I was able to bring forward certain evidence bearing on the important subject of the storage and preservation of vaccine lymph, as also on the relationship of variola and vaccinia, so far as microscopic and bacterioscopic examination of lymph can help us. These two subjects I desire particularly to consider on the present occasion.

It is a matter of common knowledge that vaccine lvmph, whether stored in the liquid state or freshly obtained from a normal vesicle, usually is found when examined bacterioscopically to contain micro-organisms of various kinds, which have been shown by Crookshank, myself, and by other observers to be for the most part mere saphrophytes. To these, therefore, I have applied the term "extraneous." The practically universal occurrence of these saphrophytic organisms in vaccine lymph, along with chance of superaddition during or after vaccination, of pathogenic organisms by agency of careless people, whether vaccinators or persons having charge of infants, have been advanced as reasons for doing away with the practice of vaccination on the grounds of the possible harmfulness of the extraneous organisms liable to be introduced into the system at the time or in the course of vaccination. This argument however, so far as the microbes usually intimately associated with lymph are concerned, loses whatever weight originally attached to it, since I have been enabled to show that by thoroughly incorporating a certain amount of chemically pure glycerine with the lymph, and afterwards storing the mixture for a time prior to use, all the ordinary saprophytes found associated with lymph are completely destroyed, as is shown by the fact that no growth arises in any of the ordinary culture media inoculated with such glycerinated lymph. This statement applies equally to the streptococcus of erysipelas, which has on occasion got admission to vaccine lymph. With reference to possible transmissions of syphilis and tuberculosis by the calf lymph, I need say nothing, seeing that it is universally admitted that the first of these two diseases is not transmissible to the calf, and that the bacillus of tuberculosis has never been discovered in vaccine lymph, bovine or human.

In glycerinated lymph we have therefore a preparation which, while even more active as vaccine than the original lymph, can, to the best of my belief, be produced absolutely free from the "extraneous" organisms which at one time or another have been isolated from fresh or stored lymph, with the possible solitary exception of bacillus subtilis, which, however, possesses no pathogenic properties. This statement, if it rested on my own authority alone, might no doubt be received with hesitation; but, seeing that since I first published in the Transactions of the Congress of Hygiene in I891 the experiments on which it is based, ample corroboration has been afforded by the fact that other observers, including Chambon, Menard, and Straus in France, Leoni in Italy, and Klein in England, have arrived at similar results. In consequence no doubt of the apparent impossibility which existed until recently of isolating any bacterium from vaccine lymph which could be regarded as specific to vaccinia, several observers have sought to prove that organisms of a somewhat higher order than bacteria might be concerned in the production of this malady and also of variola. In this connection it may be mentioned that bodies believed to be of the nature of psorosperms or sporozoa have been described as existing in the vaccine vesicle by Guarnieri and Pfeiffer on the Continent, and by Ruffer and Jackson Clarke in this country; but as to this I was taken to task last year by Ruffer for mentioning the organisms described by Guarnieri and himself in the same category with those, the occurrence of which had been put on record by Pfeiffer and Jackson Clarke. As, however, the last-named gentleman is to bring forward a paper giving the result of his researches, I do not propose to deal further with this subject on the present occasion except to put on record the joint opinion of Dr. Klein and myself to the effect that whatever may be the exact nature of these so-called sporozoa, they have not yet been proved to have any causal relationship with the diseases in question.

On the other hand, as I stated last year, Dr. Klein and I have concurrently, though quite independently, shown that in specially stained specimens of vaccine lymph, taken at a period antecedent to full maturity of the vesicles, minute bacilli can be demonstrated, often in considerable numbers, and in practically pure culture. These bacilli cannot be found or only with difficulty, in mature lymph, for the reason probably that they have by then given place to spores.

For some time it appeared impossible to obtain further evidence as to the rôle of these bacilli, as they altogether refused to grow on any of the ordinary culture media, and under either aërobic or anaërobic conditions, though this very fact obviously tends to show that they are not of a merely saprophytic nature. During the past year, however, certain experiments which I have devised appear to prove that it is possible to obtain a growth of these bacilli in a particular culture medium, and even to carry on such growth in secondary cultivation. The experiments which have been 
instituted in this direction are at present but few in number, and it is quite evident that the exact conditions essential to success have yet to be determined, but nevertheless I think that the subject is of sufficient interest to.justify me in bringing to your notice the results that so far I have obtained in this sense.

As it seemed to me, the desideratum for the purpose of inducing growth of the supposititious micro-organisms was a medium which had not been artificially sterilised, which a priori should afford the necessary pabulum, and which at the same time-whether it was to be considered aërobic or anaërobic-was secure in its circumstances against contamination. Hence the choice appeared to lie between the intraperitoneal fluids of one or another living animal and the hen's egg, which has already been made use of as an alternative culture medium.

As regards intraperitoneal fluid I foresaw difficulty, in that it is apt to contain leucocytes, which might easily vitiate experiment, and accordingly I adopted the hen's egg as a matter of first instance. I had no experience to guide me as to the best method of carrying out the inoculation of the egg, or as to the period of incubation best suited for the object I had in view, and my attempts for a time, therefore, met with but small success. My inoculations were carried out by first cleansing the surface of the egg with corrosive sublimate solution and afterwards with alcohol, and then breaking a small hole in the shell by means of a sterilised needle and forceps. Through the opening thus made I mixed the yolk and albumen of the egg by means of a platinum needle, and then carried out the inoculation by means of a glass capillary pipette, which had recently been drawn out in the blowpipe flame. For the purpose of such inoculations I employed variolous crusts obtained from the small-pox ships of the Metropolitan Asylums Board. These crusts were rubbed up in a small glass mortar with a minimal quantity of sterilised normal saline solution. Of the resulting creamy emulsion a drop or two was then taken up into the glass pipette and the inoculation carried out. Finally, the small hole in the egg was closed up, either by means of a small pledget of sterilised cotton-wool soaked in collodion or with melted sealing-wax. The eggs inoculated in this manner were then placed on beds of cotton-wool and kept in the incubator at a temperature of $37^{\circ} \mathrm{C}$. Here they were left for varying periods of time, a month being the period which was eventually found to be that which was most desirable.

On opening an egg thus treated I found that in its interior was contained a creamy material, which in great part had replaced the ordinary egg contents. This material examined in coverglass specimens appeared to contain a pure culture of one organism only, namely, a bacillus which morphologically was not to be distinguished from the bacillus which $I$ have previously described as to be found in early vaccine lymph.

My next step was to attempt to grow it in subculture and to make observation also of the effect of its direct trans ference by cutaneous inoculation into the calf. As regards attempts at subculture in all the media most usually employed for such a purpose, including gelatine, sugar gelatine, agar, glycerine agar, sugar agar, and serum, these failed as subculture of the bacilli contained in vaccine lymph had always failed when the ordinary culture media are adopted, but with the calf it was different.

\section{Calf Experiments.}

Experiment I.-On March 26th, I895, Calf A. was inoculated by eighteen incisions (fifteen on the abdomen and three on the scrotum) with a small quantity of the creamy emulsion contained in Egg No. 5, which had been incubated for one month after inoculation with small-pox crusts, as already described. On March 3 oth scrapings were taken from the vesicula points which had appeared in the line of two of the abdominal incisions, and also from one of the incisions on the scrotum which had become slightly raised and red. With material thus obtained Calf B. was on the same day inoculated by six linear incisions on a specially shaved area on the abdomen. Three incisions were inoculated with scrapings taken from the vesicular points, and three with scrapings from the in cision on the scrotum of Calf $A$. Three days later (on April 2nd) all six insertions appeared to be taking, though eventually one died away. On April 4 th the calf was photo- graphed, and on the same day a child was vaccinated from it by Dr. Cory. This child I have since seen. It has five good marks, and the mother told me that the vaccination "did splendidly," and that she had had no trouble with the child.

Experiment II.-On March 3oth, I895, Calf C. was inoculated on the right thigh in twenty-four insertions with emulsion from Egg No. 5, which had been kept in a pipette since the first calf of the series was done. By April and three small vesicular points had appeared in the line of as many of the incisions. With scrapings from these I vaccinated myself, and Dr. Cory vaccinated a child, aged 3 months. The process in my own case had aborted by the fourth day, but the child "took" quite normally, and had, when inspected on the eighth day, five good vesicles with very little areola.

On April 2nd, also, Calf D. was inoculated from the small vesicles on Calf $\mathrm{C}$. in ten incisions on a special area of the abdomen. These all showed, on the fifth day, vesicles not to be distinguished from those of like age arising from the use of current lymph on the same animal.

Experiment III.-On May 7 th, I895, Calf E. was inoculated on a special shaved portion of the abdomen, in twelve incisions from contents of Egg No. 5, taken from a pipette on the previous day. On May 14th six small vesicles, which had appeared in as many of the lines of incision, were clamped and scraped, and the material thus obtained was employed on the same date for the inoculation of Calf $F$, in twelve insertions. On May I 8 th six of these insertions had taken well. On May 2rst eleven out of these twelve insertions showed a wide crust surrounded by a vesicular ring. The width of the crust was apparently due to the calf having been able to lick the places, as its collar had been removed by mistake on May 18th. Two of the more markedly vesicular of these "places" were clamped and scraped, and with the material thus obtained Calf G. was inoculated by twenty insertions on a special area of the abdomen.

By May 28th all the twenty insertions made had "taken," and no appreciable difference could be distinguished between the vesicles which had arisen on the site of these incisions and on those which at the same date had been inoculated with current lymph. This calf was twice photographed by Mr. Kent.

From Calf G. Calf H. was inoculated on May 25th ; and, further, this strain of variola vaccine, derived in the first instance from the egg culture, was carried on through two additional removes in the calf, after which it was not further cultivated.

On May 28th, r895, lymph taken from vesicles at the third remove from the egg culture, were in my absence employed by Dr. Cory for the vaccination of a large number of children at the Animal Vaccine Establishment, owing to the fact that for some reason or other the current strain of lymph had failed to give good results in the two calves which had been vaccinated according to the usual routine. All these children showed excellent marks when inspected a week later, and several of them were photographed by Mr. Kent.

Interesting, and indeed satisfactory, as are these experiments from my point of view, I do not claim for them demonstration of the practicability of growing and multiplying outside the animal body, in artificial media, the particular cause of small-pox, or of converting the latter by my processes into the essential cause of vaccinia. And for this reason: these experiments of mine on the bovine animal had to be conducted, at any rate in this preliminary stage, at Lamb's Conduit Street, and the subjects of them had to be calves that on each occasion of experiment by me, were at the same time vaccinated in some part of their bodies with the calf lymph current at this station. It is true that every precaution was taken in the way of sterilising instruments and cleansing operation tables, in selecting a given and clearly defined area on each animal for insertion of my lymph, and for keeping the incisions made by me protected as far as possible against later accidental mediate inoculation with lymph from some part of the same or other calf into which Animal Vaccine Establishment material had been inserted. Nevertheless, it is possible that in spite of all precautions, accidental inoculation of my incisions with Animal Vaccine Establishment lymph may have occurred; and further, I am not able to deny that the 
fact of simultaneous inoculation of the calves with my egg culture and with ordinary vaccine lymph may not have, in some occult way, influenced the manifestation witnessed on the areas wherein my egg lymph was inserted. So of course these experiments have to be repeated, and are indeed in course of repetition. They involve repetition of the process from its very beginning, that is, direct inoculation of the hen's egg with crusts of variola, with calf lymph, or with human lymph ; tests by subculture in ordinary media, and in the egg, of any pure growth of microbe thus obtained, and inoculation of the calf elsewhere than at Lamb's Conduit Street, both with the primary egg cultures and with any successful subcultures in the egg, or in other media.

Should, then, further investigation confirm the results obtained in the preliminary experiments that I have described, it is possible that we may eventually be enabled to elaborate a method of specific treatment of individuals actually suffering attack by small-pox, somewhat comparable to that discovered by Behring in reference to diphtheria. Should this forecast be justified in the event, some such method would form a fitting complement to the process of preventive vaccination which now for well nigh a century has stood the test of time and of experience.

Dr. Edward Haughton (Upper Norwood) alluded to the scarcity of works on variola of late years, or of reports of post-mortem examinations of variola cases, but thought that there was sufficient basis for a just conclusion in the researches of Goze and Feltz, Oesterlen, Niemeyer, and other foreign pathologists. According to these authorities, the post-mortem appearances were extensive, especially in the kidneys and suprarenal bodies, and the time required for their production was too long to be brought within the limits of conventional infection. The phenomena of the disease corresponded with the idea that urea and carbonic acid were in excess in the blood, and that the saline constituents were diminished in variola. It was a disease of septic poisoning, and vaccinia was of the same character, and had no scientific basis for its use. Sanitary conditions and meteorological were the keys necessary for understanding the phenomena of small-pox, which originated in bad hygienic conditions without any necessary previous infection. The engorgement of the lymphatic system constituted the temporary so-called "protection" which took place after various inoculations, but from its very nature could not be permanent.

Mr. Denham (Dublin) said there was no doubt that for the purpose of propagating vaccinia from calf to calf animals approaching puberty should be avoided. This especially holds good with heifers. He was quite convinced that when proper precautions were taken all the micro-organisms found in animal vaccine were extravenous; particles of hair were a great source of trouble. Professor McWeeney had in a large series of laboratory experiments satisfied himself on this point. It was highly important that all experiments made with both vaccine matter and variolous matter should be made in a laboratory entirely apart from any public vaccine station.

Dr. C. RENNER said that one of the merits of these beautiful experiments of Dr. Copeman was the supply of an additional link in proof of the identity of variola and vaccinia by which their undoubted mutual antagonism was explained. The experiment of inoculating calves with variola lymph had been so often successful as to be conclusive. The bacteriological test afforded an additional welcome proof. The only question was about the nature of the change. Was it a reduction of virulence, a culture which might be obtained by time, various admixtures, or, on the other hand, a definite permanent change? They had certainly not yet succeeded in reversing the process. Fortunately for practice he could certainly bear out the purification of the vaccine virus by being kept for some time in pure glycerine before using it, and the bewildering number of micro-organisms which had from time to time been found in vaccine were due to extraneous impurities which were eliminated by keeping in glycerine. As a result, he had never since adopting this practice heard complaints of bad arms so called, that is, undue inflammation; on the contrary, he had often been struck by the entire absence of an areola. He had also made cultures on eggs and different substances, and observed a diplococcus, but had not as yet been so fortunate as to succeed in successfully inoculating animals with the same.

Dr. C. R. DRYsDale expressed himself as much gratified by the fact that Dr. Copeman had so successfully repeated the experiments of Ceely and Badcock, and set the matter so completely at rest. He had, ever since conversations with $\mathrm{Mr}$. Ceely and Mr. Badcock some ten years ago, been convinced that vaccinia was simply a modified form of variola occurring in the bovine species. It was fortunate that calves were more easily inoculable than cows, for in future there would be less difficulty than there had been formerly to make this question plain to the whole world. Vaccine then was merely modified variola, and acts just as an attack of variola, however weak, will protect against a powerful attack. Now that this point was cleared up, it would in the future be impossible to speak of the difficulty of saying what vaccine is. Dr. Drysdale called attention to the fact that in Berlin it was now illegal to use humanised lymph, and he thought that we in this country should endeavour to imitate that practice of Berlin in London, and also gradually to introduce the German ideal of vaccination accompanied by revaccination at the age of 12 years, a practice which has entirely got rid of variola from Germany, whilst this disease still lingers in the United Kingdom and France, owing to the fact that, after about 15 years of age, even well vaccinated patients might be occasionally infected with small-pox.

\section{ANTERIOR COLPOTOMY. ${ }^{1}$}

By A. MARTIN, M.D.,

Docent in the University of Berlin.

The more the results of modern gynæcology entitle us to undertake a surgical treatment of many organic diseases of women which do not prove amenable to medical treatment, the more we must feel bound to adopt conservative measures wherever we feel able to preserve female organs without endangering the patient's prospects.

The same conservatism must rule with reference to those cicatrices which are disposed to morbid transformation, and to endanger further on the general comfort of our patients. Undoubtedly the latter remark points particularly to abdominal section. In full appreciation of the great benefit and the high importance of this operation, we must recognise that the cicatrices of abdominal section prove to be very distressing to many women. Even when healing has been absolutely undisturbed, and when the result is a most satisfactory one in regard to the disease which required the operation, we see resulting serious discomfort from these abdominal cicatrices under different circumstances-as pregnancy, general want of strength, neurasthenia, physiological changes. of the tissues at the climacteric period, which may prove dangerous to the abdominal cicatrix, not to mention those cases in which the defective tone of the tissues, and unwise behaviour of the patient during convalescence, or last, not least, wound infection results in an abdominal abscess.

Considering this we must derive great satisfaction from seeing that within the last years operative gynæcology has applied renewed and remarkably successful attention to vaginal operations. Amongst these there is found one which has as yet only a limited application, and which ought to be given the first place in vaginal technique-anterior colpotomy.

The anatomical conformation of the pelvis offers an entrance into the abdominal cavity through the anterior fornix which is easy and at the same time safe. There we do not meet any important pelvic organs; there we penetrate that territory in which the anastomoses of the lateral vessels are not accompanied by any large arteries or veins.

Experience proves that the bladder can be separated with out difficulty from the cervix uteri, that the bladder can be pushed up out of the way, after which the peritoneum can be opened easily and with all surgical security, as the fold of the peritoneum can be freely exposed to our view. The ureters are beyond reach as they lie at the side of the cervix, and are pushed up with the bladder. The entire anterior surface of the uterus is exposed, after which the fundus can 1 Reid in the Section of Obstetrics and Gynæcology at the Annual Meet ing of t'le Blitish Medica Association held in London, July-tugust, 1895. 